\title{
Validation of the Emotiv EPOC® EEG gaming system for measuring research quality auditory ERPs
}

BACKGROUND: Auditory event-related potentials (ERPs) have proved useful in investigating the role of auditory processing in cognitive disorders such as developmental dyslexia, specific language impairment (SLI), attention deficit hyperactivity disorder (ADHD), schizophrenia, and autism. However, laboratory recordings of auditory ERPs can be lengthy, uncomfortable, or threatening for some participants - particularly children. Recently, a commercial gaming electroencephalography (EEG) system has been developed that is portable, inexpensive, and easy to set up. In this study we tested if auditory ERPs using a gaming EEG system (Emotiv EPOC®, www.emotiv.com ) were equivalent to those measured by a widely-used, laboratory-based, research EEG system (Neuroscan). METHODS: We simultaneously recorded EEGs with the research and gaming EEG systems, whilst presenting 21 adults with 566 standard $(1000 \mathrm{~Hz})$ and 100 deviant $(1200 \mathrm{~Hz})$ tones under passive (non-attended) and active (attended) conditions. The onset of each tone was marked in the EEGs using parallel port pulse (Neuroscan) or a stimulus-generated electrical pulse injected into the $\mathrm{O} 1$ and $\mathrm{O} 2$ channels (Emotiv EPOC®). These markers were used to calculate research and gaming EEG system late auditory ERPs (P1, N1, P2, N2, and P3 peaks) and the mismatch negativity (MMN) in active and passive listening conditions for each participant. RESULTS: Analyses were restricted to frontal sites as these are most commonly reported in auditory ERP research. Intra-class correlations (ICCs) indicated that the morphology of the research and gaming EEG system late auditory ERP waveforms were similar across all participants, but that the research and gaming EEG system MMN waveforms were only similar for participants with non-noisy MMN waveforms ( $N=11$ out of 21). Peak amplitude and latency measures revealed no significant differences between the size or the timing of the auditory P1, N1, P2, N2, P3, and MMN peaks. CONCLUSIONS: Our findings suggest that the gaming EEG system may prove a valid alternative to laboratory ERP systems for recording reliable late auditory ERPs (P1, N1, P2, N2, and the P3) over the frontal cortices. In the future, the gaming EEG system may also prove useful for measuring less reliable ERPs, such as the MMN, if the reliability of such ERPs can be boosted to the same level as late auditory ERPs. 
1 Authors:

2 Nicholas A Badcock

3 ARC Centre of Excellence in Cognition and its Disorders, Macquarie University

4 Sydney, NSW, Australia

5 Petroula Mousikou

6 ARC Centre of Excellence in Cognition and its Disorders, Macquarie University

7 Sydney, NSW, Australia

8 Department of Psychology, Royal Holloway, University of London

9 London, United Kingdom

10 Yatin Mahajan

11 ARC Centre of Excellence in Cognition and its Disorders, Macquarie University

12 Sydney, NSW, Australia

13 Peter de Lissa

14 ARC Centre of Excellence in Cognition and its Disorders, Macquarie University

15 Sydney, NSW, Australia

16 Johnson Thie

17 School of Electrical and Information Engineering, University of Sydney

18 Sydney, NSW, Australia

19 Genevieve McArthur

20 ARC Centre of Excellence in Cognition and its Disorders, Macquarie University

21 Sydney, NSW, Australia 
22 Corresponding Author:

23 Name: Nicholas A Badcock

24 Address:

25 ARC Centre of Excellence in Cognition and its Disorders

26 Macquarie University

27 Sydney, NSW

28 Australia, 2109

29 Phone number: + 61298504067

30 e-mail: nicholas.badcock@mq.edu.au 


\section{Introduction}

32 An auditory event-related potential (ERP) reflects the average electrical response of a large

33 groups of brain cells in response to a particular sound (e.g., a high-pitched tone). This

34 electrical activity can be measured at the scalp using electrodes. The first three positive peaks

35 in an ERP waveform are often referred to as the P1 and P2 and P3 (also referred to as the

36 P100, P200, and P300), and the first two negative peaks are often called the N1 and N2 (N100

37 and N200, see Fig. 2, F3 panels). These "late auditory ERPs" are thought to be generated by

38 neurons (i.e., brain cells) that process the physical features of sensory stimuli, and neurons

39 involved in the detection, classification, and inhibition of stimuli (Key, Dove \& Maguire

40 2005). The auditory ERP waveform is therefore considered to reflect post-synaptic electrical

41 activity predominantly in the primary and secondary auditory cortices (Oades, Zerbin \&

42 Dittmann-Balcar 1995; Tonnquist-Uhlén et al. 1996).

43 One advantage of auditory ERPs is that it is possible to measure them passively, without a

44 listener's attention; for example, whilst participants watch their favourite DVD. The

45 undemanding nature of passive auditory ERPs has made them a popular tool for measuring

46 auditory processing in inattentive listeners, such as children or adults with cognitive disorders

47 such as developmental dyslexia (McArthur, Atkinson \& Ellis 2009), specific language

48 impairment (Barry et al. 2008), autism (McPartland et al. 2004), attention-deficit

49 hyperactivity disorder (Taylor et al. 1997), and schizophrenia (Todd, Michie \& Jablensky

50 2003). One ERP component commonly measured in these populations is mismatch negativity

51 (MMN). This is calculated by subtracting a late auditory ERP to a rare “deviant” sound (e.g.,

52 high tone) from a late auditory ERP to a frequent "standard” sound (e.g., a low tone; see Fig.

53 3). This ERP is traditionally thought to reflect pre-attentive memory and auditory

54 discrimination (Näätänen 1992). However, recent research suggests that it may reflect N1

55 activity related to new afferent neuronal activity (May \& Tiitinen 2010). 
56 A limitation of auditory ERPs is that they are typically measured in an experimental

57 laboratory full of medical-looking equipment, which can be frightening for some people, such

58 as children or adults with cognitive disorders. Further, it can take an experimenter 30-40

59 minutes to place 32 electrodes on a participant's scalp, making an ERP measurement session

60 lengthy. Another limitation of ERPs is that many ERP electrode caps use a thick gel to create

61 a connection between the scalp and each electrode. At the end of a session, a person is left

62 with clumps of gel throughout their hair that can only be properly removed by thoroughly

63 washing the entire head.

64 In recent times, the commercial computer gaming industry has come up with a tantalizing

65 solution to these problems: wireless EEG systems (Emotiv EPOC®, Imec's wireless EEG

66 headset, NeuroFocus Mynd ${ }^{\mathrm{TM}}$, Neurokeeper's headset, NeuroSky Mindwave $\left.{ }^{\circledR}\right)$. These

67 “gaming EEG systems" use EEG activity to control the movement of characters or objects in

68 games via headsets that comprise a small array of sensors that (1) are wirelessly connected to

69 software that runs on a laptop (so no need for an expensive laboratory); (2) require little

70 adjustment of electrodes (so no need for long electrode placement procedures); and (3)

71 typically use small cotton pads that are soaked in saline solution to connect each electrode to

72 the scalp (so no need for messy gel, and hence no need for head washing).

73 A handful of researchers have tested the validity of using gaming EEG systems as research

74 tools. Work by Thie, Kilstorner and Graham (2012) and Debener et al. (2012) suggests that

75 these systems are valid tools for measuring visual evoked potentials and EEG activity when

76 walking outside a laboratory. However, to our knowledge, no study has validated the use of a

77 gaming EEG system for measuring ERPs, perhaps because this requires a physical

78 modification of the headset to insert stimulus markers into an EEG (see Equipment section

79 below). Thus, the aim of the current study was to test the validity of one gaming EEG system

80 as a measure of auditory ERPs, in an endeavor to minimize the stress associated with ERP 
81 measurement for some people, and allow the measurement of ERPs outside of laboratory

82 settings (e.g., schools and audiologists’ practices).

\section{Materials and Methods}

\section{Participants}

85 The Macquarie University Human Research Ethics Committee approved the methods used to

86 test participants (Ethics Ref: 5201200658). All participants gave written informed consent to

87 be involved in the research. Twenty-one typical adults (12 female and 9 male) were involved

88 in this study. The mean age of the participants was 31 years ( $\mathrm{SD}=5.3$ years). Individuals had

89 normal or corrected-to-normal vision and no history of auditory-related problems.

\section{Stimuli}

91 Stimuli were delivered to participants binaurally at a comfortable listening volume through

92 Phillips SHS4700/37 ear-clip headphones fixed to the gaming EEG system headset (see Fig. 1

93 for a diagram), using Presentation (version 16; Neurobehavioral System Inc.). There were two

94 blocks of stimuli. Both blocks comprised 566 standard tones (175-ms 1000-Hz pure tones

95 with a 10-ms rise and fall time; 85\% of trials) and 100 deviant tones (175-ms 1200-Hz pure

96 tones with a 10-ms rise and fall time; $15 \%$ of trials). After 3 standard tones (i.e., first 3 trials),

97 the presentation of the deviant tones was randomly separated by 3 to 35 standard tones. The

98 stimuli were separated by a jittered stimulus-onset synchrony (SOA) of 0.9-1.1 s to minimize

99 the ERP related to the anticipation of a stimulus.

100 The first block of stimuli was presented in a "passive condition”, in which participants were

101 instructed to watch a silent video and ignore the tones coming through the ear-clips. The

102 second block of stimuli was presented in an "active condition", in which participants were

103 asked to count the deviant (higher) tones whilst watching the silent video. They were told that

104 they would be asked to report the total number of deviant tones at the end of the session. 


\section{Equipment}

106 The Neuroscan system (version 4.3, hereafter referred to as the research EEG system) was

107 connected to an EEG electrode cap that comprised 16 sintered Ag-AgCl electrodes that were

108 sewn into a material cap according to the International 10-20 system (Easy Cap). We

109 recorded EEGs from 16 sites: F3, F7, FC4, FT7, T7, P7, O1, O2, P8, T8, FT8, FC4, F8, F4,

110 M1, and M2. The left mastoid (M1) served as online reference, which left us with 15

111 electrode sites (i.e., EEG channels). Vertical eye movements (VEOG) were measured with

112 electrodes placed above and below the left eye. Horizontal eye movements (HEOG) were

113 recorded using electrodes placed on the outer canthi of each eye. The ground electrode was

114 positioned between FPz and Fz. The online EEG was sampled at $1000 \mathrm{~Hz}$ with an online

115 bandpass filter from $1 \mathrm{~Hz}$ to $100 \mathrm{~Hz}$. During offline processing, the research EEG system data

116 was downsampled (using EEGLAB; Delorme \& Makeig 2004) to $128 \mathrm{~Hz}$ to make it

117 compatible with the gaming EEG system sample rate. The onset of each stimulus was marked

118 in the EEG using parallel port pulses.

119 The gaming EEG system (i.e., Emotiv EPOC®) used gold-plated contact-sensors that were

120 fixed to flexible plastic arms of a wireless headset (see Fig. 1). The headset included 16 sites, 121 aligned with the 10-20 system: AF3, F7, F3, FC5, T7, P7, O1, O2, P8, T8, FC6, F4, F8, FC4, $122 \mathrm{M} 1$, and M2. One mastoid (M1) sensor acted as a ground reference point to which the voltage 123 of all other sensors was compared. The other mastoid (M2) was a feed-forward reference that 124 reduced external electrical interference. The signals from the other 14 scalp sites (channels)

125 were high-pass filtered with a $0.16 \mathrm{~Hz}$ cut-off, pre-amplified and low-pass filtered at an $83 \mathrm{~Hz}$ 126 cut-off. The analogue signals were then digitised at $2048 \mathrm{~Hz}$. The digitised signal was filtered 127 using a $5^{\text {th }}$-order sinc notch filter (50 to $60 \mathrm{~Hz}$ ), low-pass filtered and down-sampled to 128 $128 \mathrm{~Hz}$ (specifications taken from the gaming EEG system web forum). The effective bandwidth 129 was 0.16 to $43 \mathrm{~Hz}$. 
130 Because the gaming EEG system was developed for measuring EEG and not ERPs, it had to

131 be modified to send markers to the EEG to indicate the onset of each stimulus. This was done

132 using a custom-made transmitter module (see below for a description) that converted each

133 tone presented by Presentation into a positive and negative electrical signal. These signals

134 were injected into the $\mathrm{O} 1$ and $\mathrm{O} 2$ channels of the gaming EEG system headset. The resulting

135 positive and negative spikes recorded in the $\mathrm{O} 1$ and $\mathrm{O} 2 \mathrm{EEGs}$ were processed offline in

136 Matlab. A between-channels difference greater than $50 \mathrm{mV}$ was coded as a stimulus onset or

137 offset. The event marker was set at a constant time interval (5 ms delay of the transmitter

138 module) prior to the point of positive and negative signal cross-over. For around $10 \%$ of

139 subjects, there were more stimulus markers than expected, which was due to participant

140 movement. These markers were removed manually. The valid stimulus markers were

141 recombined with the EEG data. The "sacrifice" of the O1 and O2 sites for the purposes of

142 stimulus marking left us with 12 gaming EEG system scalp electrode sites.

143 The custom-made transmitter module consists of two parts: audio tone detector and pulse

144 generator. The audio tone detector is connected to the headphone output of the Presentation

145 computer. Upon detecting an audio tone, it activates the pulse generator. The pulse generator

146 in turn generates a pulse to $\mathrm{O} 1$ while $\mathrm{O} 2$ is treated as the isolated ground. The amplitude of

147 the pulse is adjustable from 30 to $300 \mu \mathrm{V}$. The pulse duration is fixed at $35 \mathrm{~ms}$. The pulse

148 generator is electrically isolated from the audio tone detector by using an opto-coupler. This

149 ensures that the subject is electrically isolated from any equipment connected to the mains,

150 which is the Presentation computer in this case.

151 Testing procedure

152 Each participant was seated in a comfortable chair $1.5 \mathrm{~m}$ away from a 13-inch laptop screen.

153 The experimenter combed the participants on the left, mid-line, and right sides of their scalp

154 firmly in order to reduce electrode impedances (Mahajan \& McArthur 2010). After the 
155 relevant areas on the face and mastoids had been cleaned, the EasyCap (connected to the

156 research EEG system) was positioned on the participant's head. A blunt metal tube mounted

157 in a syringe was then used to inject conductive gel into the small gap that separated each

158 electrode from the participant's scalp. The blunt tube was circled and gently rocked back and

159 forth against the scalp 3 to 4 times before injecting the gel. This took around 30-45 minutes

160 depending on the participant's scalp conductivity.

161 Once all scalp electrodes had been filled with gel, the impedances of each electrode were

162 measured using Neuroscan Synamps ${ }^{2}$ acquisition system and the Scan software (Scan 4.3).

163 Further adjustments were made to each electrode until the impedance for all electrodes was

164 less than 5 kOhms. The gaming EEG system headset was then fitted on top of Easy Cap. The

165 gaming EEG system sensors were placed on the head through custom slits made in the

166 EasyCap (see Fig. 1). The custom slits were made by fitting the gaming EEG system headset

167 over the EasyCap and making incisions where the gaming EEG system sensors fell. Where

168 the system positions overlapped, slits were made directly adjacent to the EasyCap electrode

169 location. The sensors were adjusted until suitable connectivity was achieved as indicated by

170 the TestBench software, which adds a small modulation to the feedforward signal, and

171 measures the size of the signal back from each channel. This procedure took 10-15 minutes.

173 The research and gaming systems recorded EEG simultaneously, as opposed to recording with

174 one system and then the other in a counterbalanced fashion, to maximise the conditions for

175 validation. Specifically, if separate recordings were made and differences were noted, it would

176 be difficult to determine if these were due to differences between the systems, or due to

177 differences between the state of the brain at different points in time (e.g., differences in level

178 of fatigue, or differences in the amount of exposure to the stimuli). 
180 The research and gaming system EEGs were processed offline using EEGLAB version

181 11.0.4.3b. Major artifacts were first excluded by eye, and then the EEG in each channel was

182 bandpass filtered from 0.1 to $30 \mathrm{~Hz}$. Eye-movements and heartbeat signals (heartbeat signals

183 were present in the research system EEG for just 5 subjects) were removed using independent

184 components analysis (ICA) in EEGLAB ('eeg_runica' function). The cleaned EEG signals in

185 each channel were then cut into epochs that started -102 ms before the onset of each stimulus

186 (0 ms), and ended $500 \mathrm{~ms}$ after the onset of the same stimulus. Each epoch was baseline

187 corrected from -102 to 0 ms. Epochs with amplitude values +/- $150 \mu \mathrm{V}$ were excluded. The

188 median number of accepted epochs in the research and gaming EEG system waveforms is

189 shown in Table 1.

\section{Creating waveforms}

191 Accepted epochs were averaged together to create a standard late ERP waveform and a

192 deviant late ERP waveform (see Fig. 2) for both active and passive conditions at each scalp

193 site for each participant. The standard late ERP waveforms in both active and passive

194 conditions were used to measure the P1, N1, P2, and N2 peaks (deviant late ERP waveforms

195 were not used because these comprised fewer epochs, and hence were less reliable than

196 standard ERP waveforms). The deviant late ERP waveform in the active condition was used

197 to represent the P1, N1, P2, and N2 peaks as well as the P3 ERP peak since the P3 is

198 enhanced by active attention to a rare or unexpected event. Note that we define ERP peaks

199 and components in line with Luck (2005). That is, P1, N1, P2, N2, and P3 ERPs are peaks,

200 and the $\mathrm{MMN}$ is a component.

201 The late auditory ERP waveforms in the passive condition were used to create one MMN

202 waveform per person. We did this by subtracting the standard late auditory ERP waveform 
203 from the deviant late auditory ERP waveform (see Fig. 3). Data from the passive condition

204 was used because directing attention away from the experimental stimuli (i.e., towards a

205 movie) minimises the impact of confounding attention-related P3a and P3b ERPs on the

206 MMN (Lang et al. 1995; Sinkkonen \& Tervaniemi 2000).

\section{Measuring waveform peaks}

208 The P1, N1, P2, and N2 ERP peaks were represented by the first two clear positive peaks (P1

209 and P2), and the first two clear negative peaks (N1 and N2) in the passive and active standard

210 late auditory ERP waveforms (see Fig. 2). For all subjects, P1 fell between 15 and 120 ms; N1

211 fell between 60 and 180 ms; P2 fell between 100 and 420 ms; and N2 fell between 170 and

212490 ms. The amplitude and latency of each peak for each participant was measured via

213 manual selection of the peaks using the EEGLAB software. Manual selection was used for all

214 peaks because automated peak classification incorrectly classified peaks in a minority of

215 individuals due to variation in individuals' waveforms. For example, the waveforms of a few

216 participants had a noisy baseline that resulted in incorrect detection of P1. In this early stage

217 of research on gaming EEG systems, we felt it wise to err on the side and caution, and thus

218 manually measured peaks for each participant individually to ensure that all latency and

219 amplitude were truly valid.

220 The P3 ERP was represented by the third positive peak in the deviant late auditory ERP

221 waveform in the active condition (see Fig. 2). The amplitude and latency of the P3 peak for

222 each participant was measured via manual selection of the peak using the EEGLAB software.

223 For all subjects, this interval fell between 210 and $420 \mathrm{~ms}$.

224 The MMN was represented by the large negative deflection in the passive MMN waveform. It

225 was estimated as the minimum voltage (i.e., a negative peak) in the interval across which the

226 MMN waveform fell below 0 (i.e., when the MMN was present). For all subjects, this interval 
227 fell between 80 to and 250 ms. However, it is noteworthy that the research and gaming EEG

228 system MMN could not be calculated for two participants, and the gaming EEG system MMN

229 could not be calculated for an additional eight participants. In these cases, the deviant late

230 ERP waveform was unexpectedly (and in some cases, drastically) more positive than the

231 standard late auditory ERP waveform.

\section{Results}

233 For ease of presentation and hence understanding, all analyses focussed on data from two

234 frontal sites in the left and right hemispheres: F3 and F4 for the research EEG system, AF3

235 and AF4 for gaming EEG system. We chose these sites because they typically register the

236 largest late auditory ERP and MMN responses, and hence are most commonly used to

237 represent these ERPs in the research literature (Bishop et al. 2007; Ponton et al. 2000). The

238 mean research and gaming EEG system late auditory ERP waveforms for standard and

239 deviant stimuli in the passive and active conditions at these sites are shown in Fig. 2.

240 Morphologically, these waveforms were all consistent with mature auditory ERPs (Bishop et

241 al. 2007; Mahajan \& McArthur 2012; Ponton et al. 2000).

\section{Waveform reliability}

244 We tested the reliability of the waveforms produced by the research and gaming EEG systems

245 using the number of epochs accepted into the standard and deviant waveforms in the passive

246 and active conditions. These are shown in Table 2.

248 Due to significant negative skews in the data, we compared the accepted number of epochs

249 for each system using Wilcoxon Signed Rank Tests and a Bonferroni corrected p-value for 250 multiple comparisons ( $\mathrm{p}=.0125)$. There were significantly fewer epochs accepted for all 
251 gaming EEG system waveforms except for the deviant waveform in the active condition.

252 However, the median values in Table 1 show that there was, in fact, very little difference in

253 the median number of epochs accepted for the research and gaming EEG systems. Further, the

254 number of accepted epochs for both systems was more than adequate. Thus, the gaming EEG

255 system produced reliable waveforms - even if slightly less reliable than the research EEG

256 system.

\section{Waveform similarity}

258 We tested the similarity of the research and gaming EEG system waveforms using intraclass 259 correlations (ICC) in line with Bishop et al. (2007). ICCs reflect the similarity of waveforms 260 in terms of morphology, amplitude, and latency. We used the ICCs to measure similarity 261 across the entire waveform (i.e., -102 ms to $500 \mathrm{~ms}$ ). The ICC values were considered 262 statistically significant if 95\% confidence intervals did not include 0.

263 Table 2 shows the ICCs between the research and gaming EEG system late auditory ERP

264 waveforms in active and passive conditions. For the ERP waveforms to the standard tones, the 265 ICCs for the active and passive waveforms ranged from 0.74 to 0.80 (depending on scalp site 266 and active or passive conditions), indicating a strong similarity between the research and 267 gaming EEG system recordings. This was supported by the 95\% confidence intervals, which 268 indicated that these ICCs were statistically significant.

269 For the ERP waveforms to the deviant tones in the active condition (including the P3 peak), 270 the ICCs were 0.77 and 0.80 for left and right frontal sites, respectively. These high ICCs 271 were statistically significant. Thus, the ERP waveforms to the deviant tones in the active 272 condition measured by the research and gaming EEG systems were similar in morphology.

273 For the MMN waveform, presented in Fig. 3, which was measured in the passive condition, 274 ICC outcomes were calculated twice. The first calculation, which included all participants, 
275 indicated that the ICCs between research and gaming EEG system MMN waveforms were 276 statistically significant $(\mathrm{ICC}=0.44)$. However, these MMN waveforms were markedly less

277 similar than the ERP waveforms to the standard and deviant tones. The second calculation,

278 which excluded participants with noisy MMN waveforms (leaving $N=11$ ), revealed a

279 stronger mean ICC between the two systems (ICC $=0.71)$. Thus, the MMN waveforms

280 measured by the two systems were similar for participants who produced reliable MMN

281 waveforms. However, reliable waveforms were not produced by half of the adults in this

282 sample.

\section{Waveform peaks}

285 The research and gaming EEG system P1, N1, P2, N2, P3, and MMN peak amplitude and 286 latency measures did not differ from a normal distribution and so were compared using paired

287 sample t-tests. Descriptive and inferential statistics for the left and right hemispheres are 288 presented in Table 3. All bar one comparison indicated no statistical difference between the 289 mean peak amplitudes and latencies for the research and gaming EEG system P1, N1, P2, and 290 N2 ERPs. The exception was the P2 latency in the active condition, which was later for 291 gaming EEG system. Further, Cohen's d measures that indexed the size of the difference 292 between the research and gaming EEG system measures were typically small for both 293 amplitude and latency in the left (0.18 and 0.44 respectively) and right (0.23 and 0.47

294 respectively) frontal sites. Interestingly, the Cohen’s d measures also revealed slightly smaller 295 amplitude differences in passive than active conditions (passive standard $=.20$ and deviant $=$. 29614 ; active standard $=.26$ and deviant $=.21$ ). 
297 In addition, condition by stimulus comparisons for the research and gaming systems showed 298 no significant differences. The descriptive and inferential statistics for these comparisons are 299 included in supplementary tables: 1 for the left hemisphere comparisons, and 2 for the right.

301 The descriptive and inferential statistics for the P3 are presented in Table 4. The research and 302 gaming EEG system P3 amplitudes and latencies were not statistically different, and the 303 differences were small (all Cohen's d < 0.24) for peak and latency measures at left and right 304 frontal sites.

305 The descriptive and inferential statistics for the MMN are presented in Table 4. Across all 306 participants, the difference between the mean amplitude for the research and gaming EEG 307 system MMNs at left of right frontal electrodes was not statistically significant. However, the 308 difference between the research and gaming EEG system MMN amplitude was noticeably 309 larger (Cohen’s $d=0.37$ ) than for other ERPs (Cohen’s d 0.18 to 0.23). Removing 10

310 participants with noisy MMN waveforms from the analysis reduced this difference to Cohen's

$311 \mathrm{~d}=0.25$. Thus, the amplitude of the research and gaming EEG system MMN components was

312 similar for participants whose MMN waveforms appeared to be reliable (i.e., uncontaminated 313 by noise).

\section{Discussion}

315 The aim of the current study was to test the validity of a gaming EEG system as an auditory 316 ERP research tool. To this end, we modified the gaming EEG headset so that it could insert

317 stimulus markers into the EEG. We then took simultaneous EEG measurements using a 318 research EEG system and a gaming EEG system in 21 adults who were presented with 666 319 standard and deviant tones under passive and active listening conditions. 
320 The analyses were restricted to the frontal sites as these register the largest late auditory ERP

321 responses and are most typically reported in the literature (Bishop et al. 2007; Ponton et al.

322 2000). We processed the EEG data offline to calculate the auditory ERP waveform to the

323 standard tones, the ERP waveform to the deviant tones in the active condition, and the MMN

324 waveform. In line with previous research, we used left and right frontal sites to represent

325 these auditory ERPs. ICCs indicated that the ERP waveforms to both the standard and deviant

326 tones were similar for the research and gaming EEG systems. This was not the case for the

327 MMN waveform for a number of participants in the study. A potential explanation for this

328 finding is the lower reliability of the MMN compared to late auditory ERP (Mahajan \&

329 McArthur 2011; McArthur, Bishop \& Proudfoot 2003): This lower reliability is likely due to

330 the reduced number of epochs used to generate the deviant waveform, against which the

331 standard waveform is subtracted, thus introducing error variance into the MMN. This

332 explanation was confirmed by the removal of participants with particularly noisy MMN

333 responses, which improved the ICC for the MMN waveform markedly. Thus, research and

334 gaming EEG system MMN waveforms were only similar for participants with reliable MMN

335 waveforms.

336 The ICCs also revealed that the deviant waveforms were less reliable in the passive $(\mathrm{M}=.63)$

337 than the active $(\mathrm{M}=.79)$ condition (see Table 2$)$. A speculative explanation for this difference

338 is movement interference. The passive condition was always completed first. With time, the

339 gel of the research EEG system dries, providing a more stable bridge from the electrode to the

340 scalp. This stability would be greatest in the active condition, completed second. Therefore,

341 subtle participant movements, not necessarily affecting the number of accepted epochs (see

342 Table 1) would have a greater influence in the passive condition. Because these movements

343 are unlikely to affect the two systems equivalently, the result would be a reduced similarity

344 between the waveforms measured with each system. This would be most evident to the 
345 deviant over the standard stimuli due to the deviant waveform being derived from fewer

346 epochs.

347 The peak amplitude and latency measures supported the ICC outcomes. In all bar one case

348 (P2 latency was later for the gaming EEG system in the active condition), there was no

349 statistically significant difference between the amplitude and latency of the P1, N1, P2, N2,

350 P3, and MMN peaks. However, there was a larger difference between the research and

351 gaming EEG system MMN amplitude measures than the late auditory ERP measures. Further,

352 the (non-significant) differences between the research and gaming EEG system late auditory

353 ERP peak measures were smaller for the passive than active condition.

\section{Conclusions}

355 Considered together, the results of this study suggest that the gaming EEG system compares

356 well with the research EEG system for reliable auditory ERPs such as the P1, N1, P2, N2, and

357 P3 measured at the frontal sites. In the future, Emotiv may also prove useful for measuring

358 less reliable ERPs, such as the MMN, if the reliability of such ERPs can be boosted to the

359 same level as late auditory ERPs. The apparent validity of the gaming EEG system for

360 measuring reliable auditory ERPs, paired with its quick and clean set-up procedure and its

361 portability, makes it a promising tool for measuring auditory processing in people from

362 special populations who are unable or unwilling to be tested in an experimental laboratory. It

363 may also open up new avenues for research since, in principle, the gaming EEG system can

364 be used anywhere (e.g., schools, homes, shopping centres, hospitals) to measure brain

365 responses in children and adults.

\section{Acknowledgements}

367 This research was supported by an ARC Centre of Excellence Grant [CE110001021] and an

368 NHMRC equipment grant. We would like to participants who volunteered their time. 
370 Barry JG, Hardiman MJ, Line E, White KB, Yasin I, Bishop DVM. 2008. Duration of Auditory Sensory Memory in Parents of Children with SLI: A Mismatch Negativity Study. Brain and Language 104:75-88.

373 Bishop DV, Hardiman M, Uwer R, von Suchodoletz W. 2007. Maturation of the long-latency 374 auditory ERP: step function changes at start and end of adolescence. Developmental Science 10:565-575.

Debener S, Minow F, Emkes R, Gandras K, de Vos M. 2012. How about taking a low-cost, small, and wireless EEG for a walk? Psychophysiology 49:1617-1621.

Delorme A, Makeig S. 2004. EEGLAB: an open source toolbox for analysis of single-trial EEG dynamics including independent component analysis. Journal of Neuroscience Methods 134:9-21.

Key APF, Dove GO, Maguire MJ. 2005. Linking Brainwaves to the Brain: An ERP Primer. 382 Developmental Neuropsychology 27:183-215.

383 Lang AH, Eerola O, Korpilahti P, Holopainen I, Salo S, Aaltonen O. 1995. Practical issues in 384 the clinical application of mismatch negativity. Ear and hearing 16:118-130.

Luck SJ. 2005. An introduction to the event-related potential technique. The MIT Press: Cambridge, MA. Journal of Neuroscience Methods 188:287-289. 
Mahajan Y, McArthur G. 2011. The effect of a movie soundtrack on auditory event-related potentials in children, adolescents, and adults. Clinical Neurophysiology 122:934-941.

Mahajan Y, McArthur G. 2012. Maturation of auditory event-related potentials across adolescence. Hearing Research 294:82-94.

May PJC, Tiitinen H. 2010. Mismatch negativity (MMN), the deviance-elicited auditory deflection, explained. Psychophysiology 47:66-122.

McArthur G, Atkinson C, Ellis D. 2009. Atypical brain responses to sounds in children with specific language and reading impairments. Developmental Science 12:768-783.

McArthur GM, Bishop DVM, Proudfoot M. 2003. Do video sounds interfere with auditory event-related potentials? Behavior research methods, instruments, \& computers: a journal of the Psychonomic Society, Inc 35:329-333.

McPartland J, Dawson G, Webb SJ, Panagiotides H, Carver LJ. 2004. Event-related brain potentials reveal anomalies in temporal processing of faces in autism spectrum disorder. Journal of Child Psychology and Psychiatry 45:1235-1245.

Näätänen R. 1992. Attention and brain function. Lawrence Erlbaum Associates, Publishers: Hillsdale, New Jersey Hove and London.

Oades RD, Zerbin D, Dittmann-Balcar A. 1995. The topography of event-related potentials in passive and active conditions of a 3-tone auditory oddball test. The International Journal of Neuroscience 81:249-264.

Ponton CW, Eggermont JJ, Kwong B, Don M. 2000. Maturation of human central auditory system activity: evidence from multi-channel evoked potentials. Clinical Neurophysiology 111:220-236. 
411 Sinkkonen J, Tervaniemi M. 2000. Towards optimal recording and analysis of the mismatch negativity. Audiology \& neuro-otology 5:235-246.

413 Taylor MJ, Sunohara GA, Khan SC, Malone MA. 1997. Parallel and serial attentional processes in ADHD: ERP evidence. Developmental Neuropsychology 13:531-539.

415 Thie J, Klistorner A, Graham SL. 2012. Biomedical signal acquisition with streaming wireless communication for recording evoked potentials. Documenta ophthalmologica. Advances in ophthalmology 125:149-159.

418 Todd J, Michie P., Jablensky A. 2003. Association between reduced duration mismatch negativity (MMN) and raised temporal discrimination thresholds in schizophrenia. Clinical Neurophysiology 114:2061-2070.

421 Tonnquist-Uhlén I, Borg E, Persson HE, Spens KE. 1996. Topography of auditory evoked cortical potentials in children with severe language impairment: the N1 component. Electroencephalography and Clinical Neurophysiology/Evoked Potentials Section 100:250-260. 


\section{Table $\mathbf{1}_{\text {(on next page) }}$}

Median number of accepted epochs for research and gaming EEG systems by condition and tone type

Median number of accepted epochs for research and gaming EEG systems by condition and tone type Median (inter-quartile range) trial numbers for the research and gaming EEG systems in each Condition (Passive versus Active listening) and for each Tone type (Standard, Deviant, and Total). Wilcoxon Signed Rank Test Z values are also presented. 


\section{EEG System}

\begin{tabular}{llccc} 
Condition & Tone & Research & Gaming & $\mathrm{Z}$ \\
\hline Passive & Standard & $564(3)$ & $558(18)$ & $3.7^{*}$ \\
& Deviant & $100(1)$ & $98(1)$ & $3.11^{*}$ \\
& Total & $663(3)$ & $657(21)$ & \\
\hline Active & Standard & $563(5)$ & $559(11)$ & $3.33^{*}$ \\
& Deviant & $100(1)$ & $98(3)$ & 2.15 \\
& Total & $663(7)$ & $658(10)$ & \\
\hline
\end{tabular}

$1 * \mathrm{p}<0.0125$ Bonferonni corrected for 4 comparisons 


\section{Table $2_{\text {(on next page) }}$}

Research versus gaming EEG system ERP and MMN waveform Intraclass Correlations

Mean intraclass correlations (ICC) and 95\% confidence intervals between waveforms simultaneously recorded with the research and gaming EEG systems for the left (F3/AF3) and right (F4/AF4)

hemispheres. ICCs are presented for the passive and active listening conditions as well as the standard and deviant tones. For the passive condition, the ICCs for the deviant minus standard waveforms, the mismatch negativity (MMN), is also presented ( $\mathrm{n}=21$ but see note a). 


\begin{tabular}{llcc}
\hline Condition & ERP & \multicolumn{2}{c}{ Hemisphere } \\
& & F3/AF3 & F4/AF4 \\
\hline Passive & Standard & $0.74(0.12)$ & $0.74(0.11)$ \\
& Deviant & $0.57(0.18)$ & $0.67(0.14)$ \\
& MMN & $0.44(0.17)$ & $0.44(0.19)$ \\
& MMN & $0.71(0.16)$ & $0.71(0.19)$ \\
\hline Active & Standard & $0.79(0.12)$ & $0.8(0.09)$ \\
& Deviant & $0.77(0.08)$ & $0.8(0.08)$ \\
\hline
\end{tabular}

1 a: $n=11$, exclusion based on manual evaluation of waveform reliability (i.e., spikes of noise 2 rather than smooth waveform) 


\section{Table 3 (on next page)}

Research versus gaming EEG system ERP peak comparisons

Descriptive (M and SD) and inferential (t and Cohen’s d) statistics for peak (P1, N1, P2, N2)

amplitude $(\boldsymbol{\mu V})$ and latency (ms) for research versus gaming EEG system comparisons in the passive and active listening conditions across both hemispheres, denoted by site $(\mathrm{n}=21)$. 


\begin{tabular}{|c|c|c|c|c|c|c|c|}
\hline \multirow[t]{2}{*}{ Condition } & \multirow[t]{2}{*}{ ERP } & \multirow[t]{2}{*}{ Measure } & \multirow[t]{2}{*}{ Site } & \multicolumn{2}{|c|}{ EEG System } & \multirow[t]{2}{*}{$\mathrm{t}$} & \multirow[t]{2}{*}{$\mathrm{d}$} \\
\hline & & & & Research & Gaming & & \\
\hline \multirow[t]{16}{*}{ Passive } & \multirow[t]{4}{*}{ P1 } & \multirow[t]{2}{*}{ Amplitude } & F3/AF3 & $0.76(0.72)$ & $0.68(1.01)$ & -0.36 & 0.10 \\
\hline & & & F4/AF4 & $0.75(0.79)$ & $0.53(1.42)$ & -0.74 & 0.19 \\
\hline & & \multirow[t]{2}{*}{ Latency } & F3/AF3 & $58.6(12.35)$ & $60.62(22.53)$ & 0.41 & 0.11 \\
\hline & & & F4/AF4 & $59.71(14.6)$ & $63.88(20.17)$ & 1.42 & 0.24 \\
\hline & \multirow[t]{4}{*}{ N1 } & \multirow[t]{2}{*}{ Amplitude } & F3/AF3 & $-3.14(1.59)$ & $-2.77(2.18)$ & 0.91 & 0.2 \\
\hline & & & F4/AF4 & $-3.36(1.71)$ & $-2.79(2.34)$ & 1.18 & 0.29 \\
\hline & & \multirow[t]{2}{*}{ Latency } & F3/AF3 & $104.86(8.1)$ & 110.85 (17.85) & 1.74 & 0.45 \\
\hline & & & F4/AF4 & $106.29(8.31)$ & $111.96(18)$ & 1.71 & 0.42 \\
\hline & \multirow[t]{4}{*}{$\mathrm{P} 2$} & \multirow[t]{2}{*}{ Amplitude } & F3/AF3 & 1.85 (1.33) & $1.36(1.31)$ & -1.55 & 0.39 \\
\hline & & & F4/AF4 & $1.87(1.42)$ & $1.47(1.53)$ & -1.16 & 0.28 \\
\hline & & \multirow[t]{2}{*}{ Latency } & F3/AF3 & $177.3(14.9)$ & 196.39 (27.98) & 3.45 & 0.88 \\
\hline & & & F4/AF4 & 176.78 (15.69) & $196.91(35.26)$ & 3.21 & 0.76 \\
\hline & \multirow[t]{4}{*}{ N2 } & \multirow[t]{2}{*}{ Amplitude } & F3/AF3 & $-1.37(1.4)$ & $-1.3(1.61)$ & 0.2 & 0.05 \\
\hline & & & F4/AF4 & $-1.34(1.48)$ & $-1.24(1.4)$ & 0.33 & 0.07 \\
\hline & & \multirow[t]{2}{*}{ Latency } & F3/AF3 & 277.95 (24.59) & 280.17 (37.08) & 0.5 & 0.07 \\
\hline & & & F4/AF4 & $278.15(28.16)$ & $284.73(41.05)$ & 0.88 & 0.19 \\
\hline \multirow[t]{16}{*}{ Active } & \multirow[t]{4}{*}{$\mathrm{P} 1$} & \multirow{2}{*}{ Amplitude } & F3/AF3 & $0.87(0.67)$ & $0.71(0.97)$ & -0.87 & 0.2 \\
\hline & & & F4/AF4 & $0.99(0.62)$ & $0.72(0.88)$ & -1.83 & 0.36 \\
\hline & & \multirow[t]{2}{*}{ Latency } & F3/AF3 & $59.64(15.18)$ & $63.81(20.5)$ & 0.83 & 0.24 \\
\hline & & & F4/AF4 & $57.23(19.94)$ & $63.16(18.72)$ & 1.84 & 0.32 \\
\hline & \multirow[t]{4}{*}{ N1 } & \multirow[t]{2}{*}{ Amplitude } & F3/AF3 & $-2.62(1.58)$ & $-2.26(1.94)$ & 1.01 & 0.21 \\
\hline & & & F4/AF4 & $-2.78(1.55)$ & $-2.18(1.69)$ & 1.75 & 0.39 \\
\hline & & \multirow[t]{2}{*}{ Latency } & F3/AF3 & $107.72(15.84)$ & 115.41 (17.73) & 1.68 & 0.47 \\
\hline & & & F4/AF4 & $108.31(18.27)$ & $115.8(18.35)$ & 2.15 & 0.42 \\
\hline & \multirow[t]{4}{*}{$\mathrm{P} 2$} & Amplitude & F3/AF3 & 2.12 (1.39) & $1.84(1.43)$ & -1.1 & 0.21 \\
\hline & & & F4/AF4 & $2.35(1.52)$ & $1.7(1.51)$ & -3.23 & 0.45 \\
\hline & & Latency & F3/AF3 & 180.17 (17.27) & $198.47(25.8)$ & $3.87 *$ & 0.86 \\
\hline & & & F4/AF4 & $183.1(20.78)$ & 201.4 (29.7) & 3.57 & 0.74 \\
\hline & N2 & Amplitude & F3/AF3 & $-0.85(1.18)$ & $-0.66(1.11)$ & 0.96 & 0.17 \\
\hline & & & F4/AF4 & $-0.71(1.13)$ & $-0.64(0.93)$ & 0.42 & 0.07 \\
\hline & & Latency & F3/AF3 & $283.23(26.61)$ & $290.65(27.56)$ & 1.8 & 0.28 \\
\hline & & & F4/AF4 & 273.52 (32.95) & $292.8(27.41)$ & 2.85 & 0.66 \\
\hline
\end{tabular}

$1 * \mathrm{p}<.0015$, Boneferonni corrected for 32 comparisons 


\section{Table 4(on next page)}

Research versus gaming EEG system P3 and MMN comparisons

Descriptive (M and SD) and inferential (t, Cohen’s d) statistics for P3 peak amplitude ( $\boldsymbol{\mu}$ V ) and latency (ms) and Mismatch Negativity (MMN) amplitude ( $\boldsymbol{\mu}$ V ) for research (F3/F4) versus gaming (AF3/AF4) EEG system comparisons ( $\mathrm{n}=21$ but see notes a and $\mathrm{b}$ ). 


\begin{tabular}{|c|c|c|c|c|c|c|}
\hline \multirow[t]{2}{*}{ ERP } & \multirow[t]{2}{*}{ Measure } & \multirow[t]{2}{*}{ Site } & \multicolumn{2}{|c|}{ EEG System } & \multirow[t]{2}{*}{$\mathrm{t}$} & \multirow[t]{2}{*}{$\mathrm{d}$} \\
\hline & & & Research & Gaming & & \\
\hline \multirow[t]{4}{*}{ P3 } & Amplitude & F3/AF3 & $3.61(3.1)$ & 4.32 (3.39) & 1.13 & 0.23 \\
\hline & & F4/AF4 & 3.48 (3.13) & $3.56(2.81)$ & 0.17 & 0.03 \\
\hline & Latency & F3/AF3 & 333.39 (52.68) & 327.14 (55.23) & -0.85 & 0.12 \\
\hline & & $\mathrm{F} 4 / \mathrm{AF} 4$ & 328.96 (49.68) & $326.1(56.01)$ & -0.46 & 0.06 \\
\hline \multirow[t]{4}{*}{$\mathrm{MMN}^{\mathrm{a}}$} & Amplitude & F3/AF3 & $-3.24(1.92)$ & $-3.17(1.71)$ & -0.22 & 0.04 \\
\hline & & $\mathrm{F} 4 / \mathrm{AF} 4$ & $-3.26(2.34)$ & $-2.82(1.68)$ & -0.97 & 0.23 \\
\hline & Latency & F3/AF3 & $159.8(24.55)$ & 149.15 (26.48) & 1.97 & 0.45 \\
\hline & & F4/AF4 & $153.41(24.02)$ & 144.18 (19.88) & 2.08 & 0.45 \\
\hline \multirow[t]{4}{*}{$\mathrm{MMN}^{\mathrm{b}}$} & Amplitude & F3/AF3 & $-3.22(2.25)$ & $-2.94(1.63)$ & -0.70 & 0.15 \\
\hline & & F4/AF4 & $-3.38(2.53)$ & $-2.69(1.55)$ & -1.48 & 0.35 \\
\hline & Latency & F3/AF3 & $163.02(37.8)$ & $145.31(23.77)$ & 1.78 & 0.59 \\
\hline & & F4/AF4 & $150.52(40.21)$ & $136.46(24.86)$ & 1.71 & 0.44 \\
\hline
\end{tabular}

1 a: $n=15$, exclusion based on missing values (i.e., incalculable due to the deviant waveform

2 being higher than standard)

3 b: $n=11$, exclusion based on manual evaluation of waveform reliability (i.e., spikes of noise

4 rather than smooth waveform) 


\section{Figure 1}

Schematic diagram of simultaneous research EEG system (Neuroscan Synamps ${ }^{2}$, in grey) and gaming EEG system (Emotiv EPOC, in black) setup.

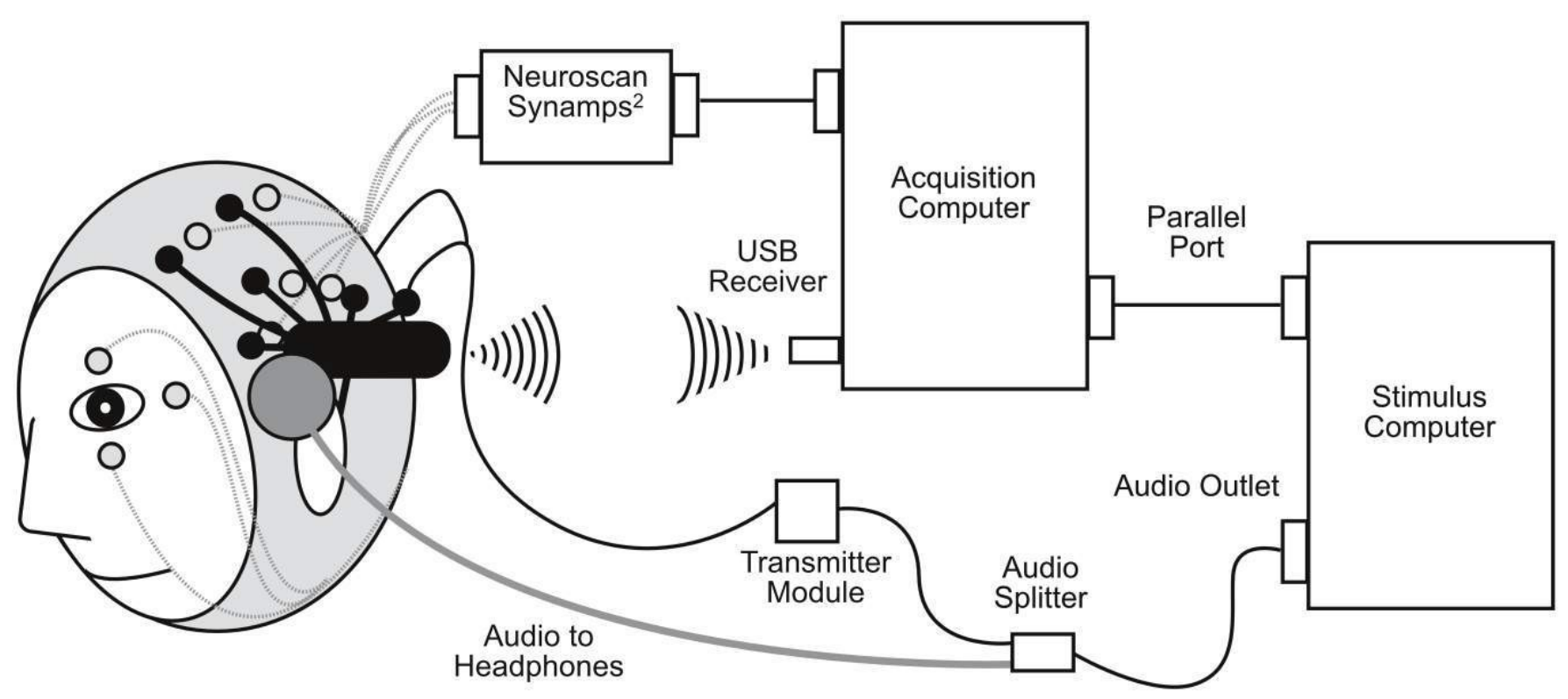




\section{Figure 2}

Research and gaming system ERP waveforms by condition, tone type, and hemisphere

Group ERP waveforms for research (left-side) and gaming (right-side) systems. All graphs display waveforms for the passive and active (counting deviant tones) listening conditions. The upper 4 graphs depict the left-hemisphere-activity (F3 and AF3) and the lower 4 graphs depict the right-hemisphere-activity (F4 and AF4). Rows 1 and 3 depict waveforms elicited by the standard tones, rows 2 and 4 depicts waveforms elicited by the deviant tones. Error waveforms (in grey) represent the standard error of the mean. 
Research
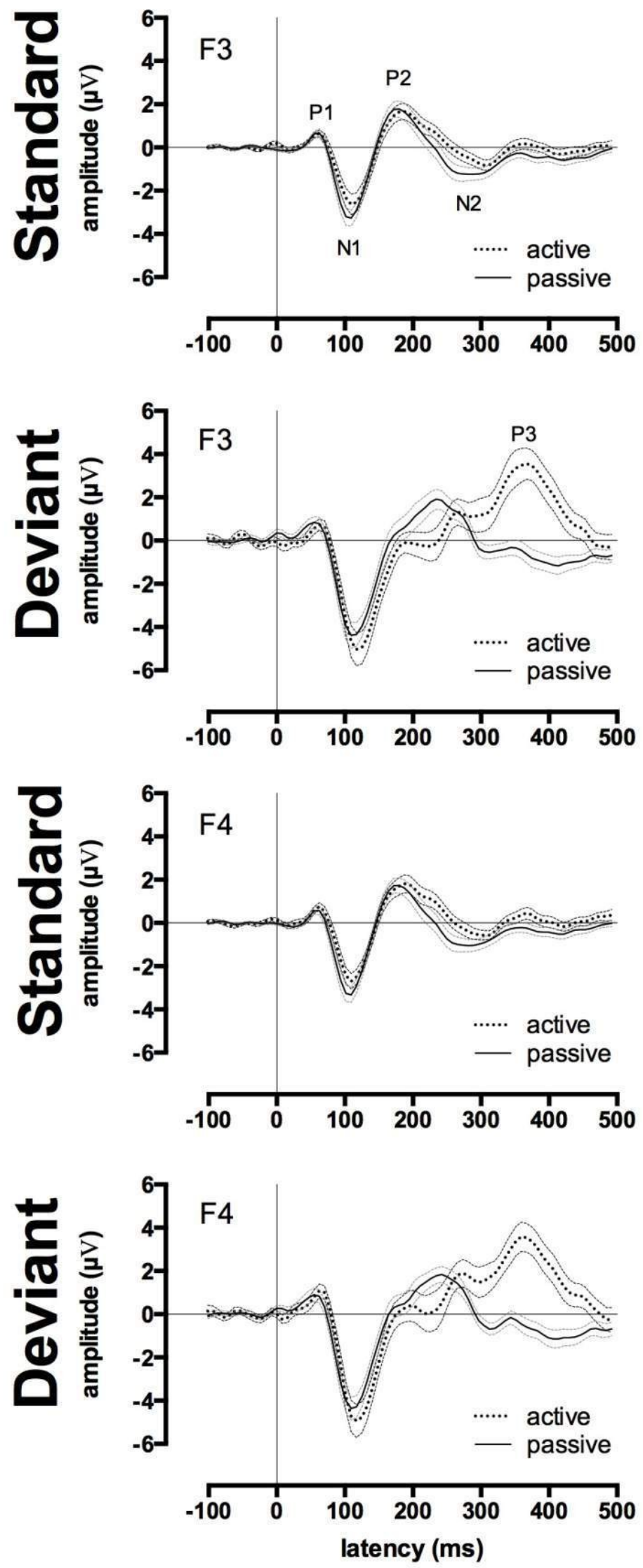

Gaming
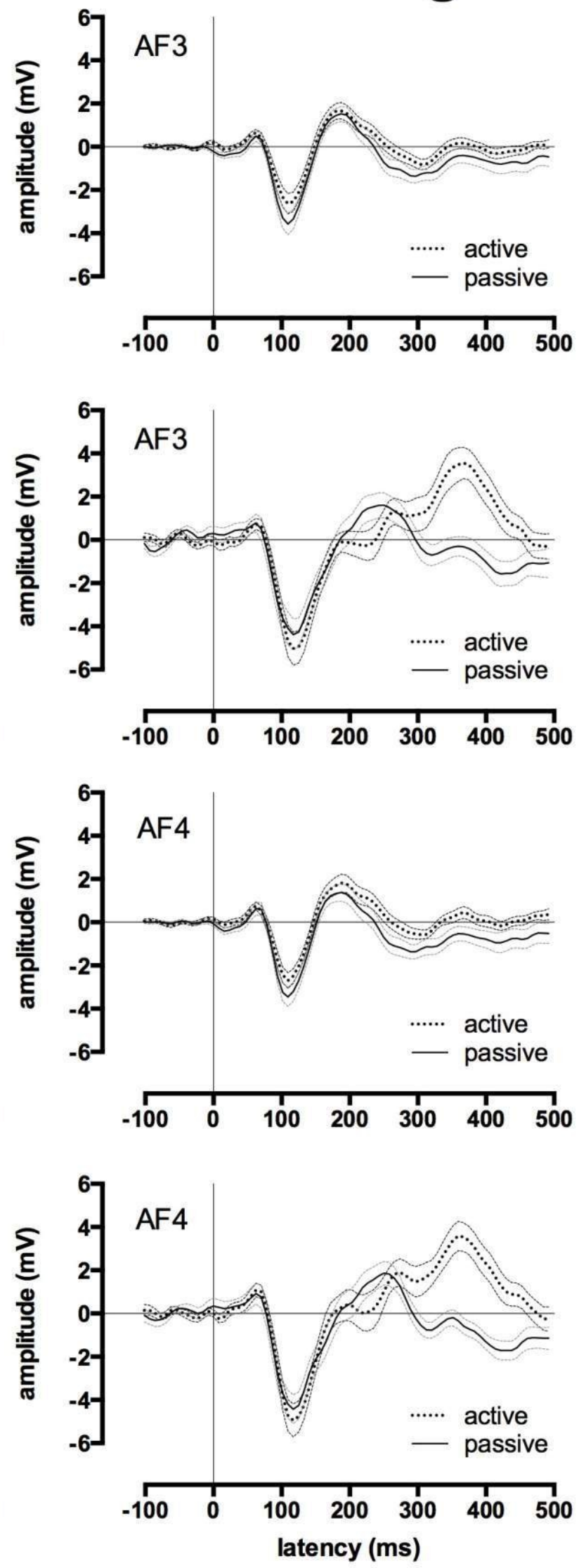


\section{Figure 3}

Research and gaming system Mismatch Negativity related waveforms by hemisphere

Group ERP and Mismatch Negativity (MMN) waveforms for research (left-side) and gaming

(right-side) systems. All graphs display waveforms for the passive listening condition. The upper 4 graphs depict the left-hemisphere-activity (F3 and AF3) and the lower 4 graphs depict the right-hemisphere-activity (F4 and AF4). Rows 1 and 3 depict waveforms elicited by the standard tones and deviant tones, rows 2 and 4 depict MMN waveforms (deviant minus standard waveforms). Error waveforms (in grey) represent the standard error of the mean. 
Research

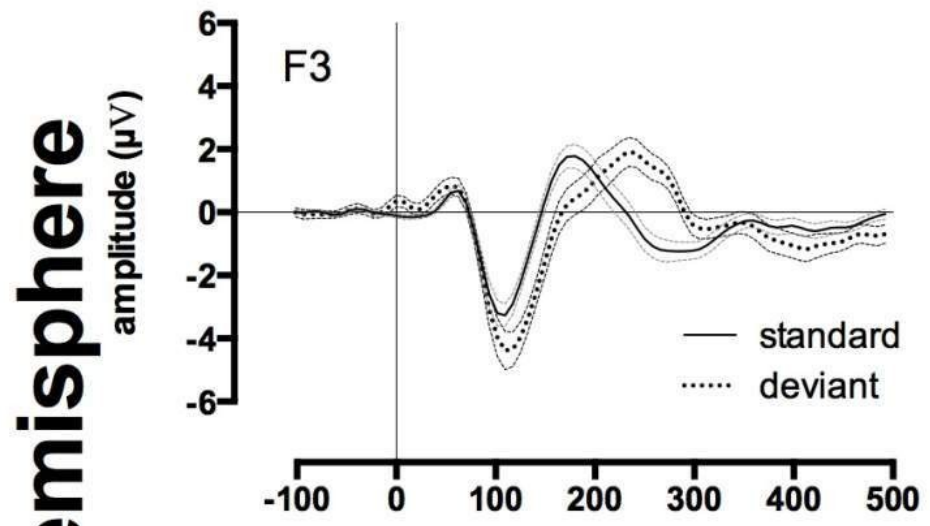

또
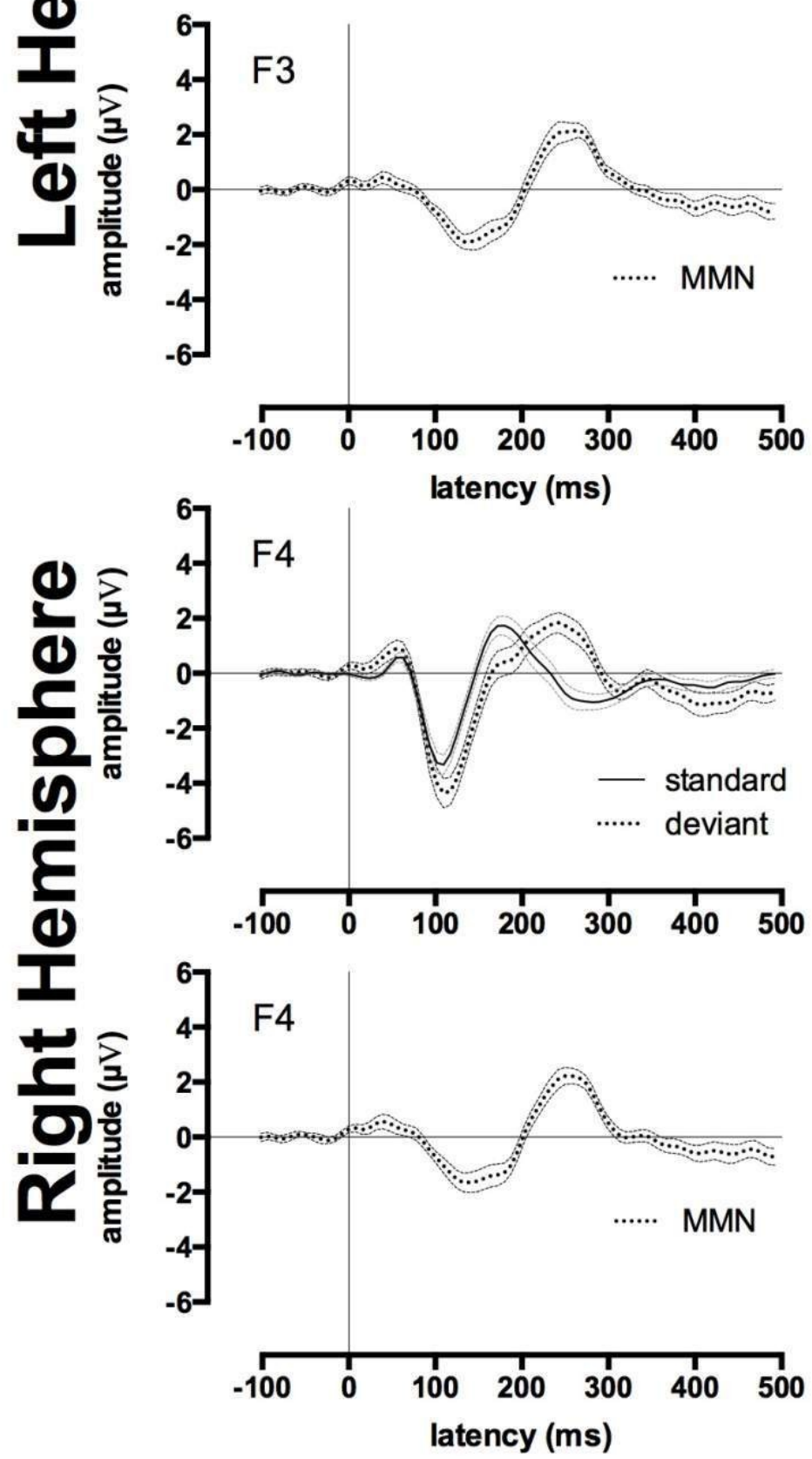

Gaming
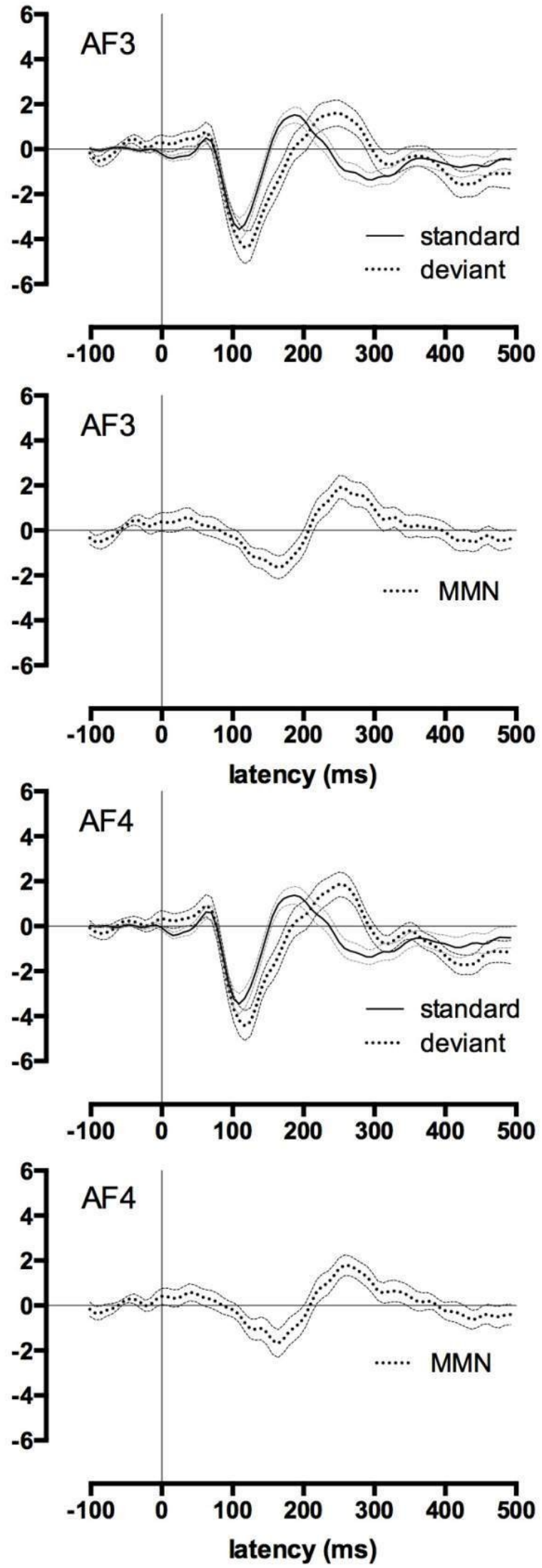